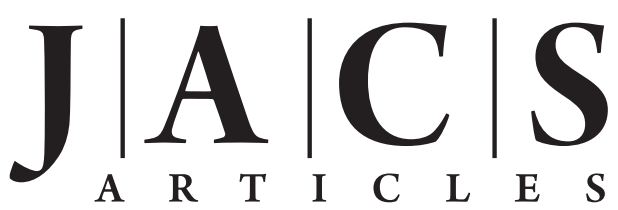

Published on Web 08/12/2009

\title{
Fluorescent Asymmetrically Cobalt-Tipped CdSe@CdS Core@Shell Nanorod Heterostructures Exhibiting Room-Temperature Ferromagnetic Behavior
}

\author{
Sasanka Deka, ${ }^{*,+,, \S}$ Andrea Falqui," Giovanni Bertoni," Claudio Sangregorio, ${ }^{\perp}$ \\ Giordano Poneti, ${ }^{\perp}$ Giovanni Morello, ${ }^{\dagger}$ Milena De Giorgi, ${ }^{\dagger}$ Cinzia Giannini, ${ }^{,}$ \\ Roberto Cingolani, ${ }^{\dagger, \|}$ Liberato Manna, ${ }^{\dagger, \|}$ and P. Davide Cozzoli ${ }^{*,+, \neq}$ \\ National Nanotechnology Laboratory of CNR-INFM, Unità di Ricerca IIT, Distretto Tecnologico \\ ISUFI, via per Arnesano km 5, I-73100 Lecce, Italy, Scuola Superiore ISUFI, Università del Salento, \\ Distretto Tecnologico ISUFI, via per Arnesano km 5, I-73100 Lecce, Italy, Fondazione Istituto \\ Italiano di Tecnologia, IIT, Via Morego 30 - 16163 Genova, Italy, UdR INSTM and Dipartimento di \\ Chimica, Università di Firenze, Via della Lastruccia 3, Sesto Fiorentino, I-50019, Italy, and \\ CNR-Istituto di Cristallografia (IC), via Amendola 122/O, I-70126, Bari, Italy
}

Received June 3, 2009; E-mail: davide.cozzoli@unile.it; ssdeka@gmail.com

\begin{abstract}
A colloidal two-step seeded-growth approach has been devised to selectively synthesize threecomponent magnetic/semiconductor hybrid nanocrystals (HNCs) with a matchstick-like profile and tunable geometric parameters. The newly developed heterostructures individually comprise a single metallic Co head connected to either apexes of one rod-shaped section made of a CdSe core eccentrically embedded in a CdS shell. The specific topological arrangement realized arises from the peculiar anisotropic reactivity of the noncentrosymmetric CdSe@CdS core@shell nanorods that have been used as substrates to seed heterogeneous nucleation of $\mathrm{Co}$ in a surfactant-free environment from an organometallic precursor. The HNCs retain appreciable fluorescent emission in spite of photoexcited charge transfer from the semiconductor to the metal domain and exhibit unusual ferromagnetic-like behavior at room temperature.
\end{abstract}

\section{Introduction}

Colloidal hybrid nanocrystals (HNCs) represent last-generation breeds of wet-chemically synthesized inorganic nanoheterostructures, in which distinct material sections are interconnected via direct bonding interfaces in elaborate onion-like or asymmetric oligomer-type configurations. ${ }^{1-4}$ The development of HNCs embodies a generic approach to advanced multicomponent nanoscale entities, whereby an increasingly higher level of structural-architectural sophistication allows enhanced and/ or diversified capabilities to be achieved by combining control over the geometry and composition of the constituent component domains with engineering of their relative spatial arrangement. ${ }^{1-4}$

The availability of complex topologically defined HNCs indeed promises to open up intriguing scenarios in many

\footnotetext{
$\dagger$ National Nanotechnology Laboratory of CNR-INFM.

† Università del Salento.

$\S$ Present Address: Istituto Italiano di Tecnologia, IIT, Via Morego 30 16163 Genova, Italy.

"Istituto Italiano di Tecnologia.

${ }^{\perp}$ Università di Firenze.

\# CNR-Istituto di Crystallografia.

(1) Cozzoli, P. D.; Pellegrino, T.; Manna, L. Chem. Soc. Rev. 2006, 35, 1195-1208.

(2) Buonsanti, R.; Casavola, M.; Caputo, G.; Cozzoli, P. D. Recent Pat. Nanotechnol. 2007, 1, 224-232.

(3) Casavola, M.; Buonsanti, R.; Caputo, G.; Cozzoli, P. D. Eur. J. Inorg. Chem. 2008, 837-854.

(4) Caputo, G.; Buonsanti, R.; Casavola, M.; Cozzoli, P. D. Synthetic strategies to multi-material hybrid nanocrystals. In Advanced WetChemical Synthetic Approaches to Inorganic Nanostructures; Cozzoli, P. D., Ed.; Transworld Research Network: Kerala, India, 2008; pp 407-453.
}

fundamental and practical fields of nanoscience. Primarily, individually processable HNCs, in which diverse material portions, each characterized by peculiar optical, magnetic, and/ or chemical functionalities, can coexist, are extremely attractive as key elements for assembling functional composite "superstructures", ${ }^{1-8}$ implementing catalytic processes, ${ }^{1-4,8-13}$ implanting a site-selective distribution of surface moieties, ${ }^{1-4,14,15}$ and enabling multimodal techniques for biomedical diagnostics/ therapeutics. ${ }^{1-4,14-16}$ Additionally, HNCs represent artificial platforms on which electronic communication effects establishing across neighboring material sections can lead to reinforced and/or tunable chemical-physical responses, or even to emer-

(5) (a) Cozzoli, P. D.; Manna, L. Nat. Mater. 2005, 4, 801-802. (b) Salant, A.; Amitay-Sadovsky, E.; Banin, U. J. Am. Chem. Soc. 2006, 128, 10006-10007.

(6) Carbone, L.; et al. Nano Lett. 2007, 7, 2942-2950.

(7) Figuerola, A.; Franchini, I. R.; Fiore, A.; Mastria, R.; Falqui, A.; Bertoni, G.; Bals, S.; Tendeloo, G. V.; Kudera, S.; Cingolani, R.; Manna, L. Adv. Mater. 2009, 21, 550-554.

(8) Elmalem, E.; Saunders, A. E.; Costi, R.; Salant, A.; Banin, U. Adv. Mater. 2008, 20, 4312-4317.

(9) Habas, S. E.; Lee, H.; Radmilovic, V.; Gabor, A.; Somorjai; Yang, P. Nat. Mater. 2007, 6, 692-697.

(10) Lee, H.; Habas, S. E.; Somorjai, G. A.; Yang, P. J. Am. Chem. Soc. 2008, 130, 5406-5407.

(11) Luo, J.; Wang, L.; Mott, D.; Njoki, P. N.; Lin, Y.; He, T.; Xu, Z.; Wanjana, B. N.; Lim, I.; Im, S.; Zhong, C.-J. Adv. Mater. 2008, 20, $4342-4347$.

(12) Joo, S. H.; Park, J. Y.; Tsung, C.-K.; Yamada, Y.; Yang, P.; Somorjai, G. A. Nat. Mater. 2009, 8, 126-131.

(13) (a) Yin, H.; Wang, C.; Zhu, H.; Overbury, S. H.; Sun, S.; Dai, S. Chem. Commun. 2008, 4357-4359. (b) Wang, C.; Daimon, H.; Sun, S. Nano Lett. 2009, 9, 1493-1496. 
gence of unprecedented properties, not otherwise accessible by any of the single components alone, or their physical mixture counterparts. In this regard, the most known examples concern HNCs based on semiconductors and/or noble metals, whereby remarkable changes in optical extinction/emission and/or electrical conduction manifest altered charge carrier localization, recombination, separation and/or plasmon-to-exciton coupling phenomena, which have implications in optoelectronic, photovoltaic, and photoelectrocatalytic applications. ${ }^{1-4,8,17-24}$ The impact of attaining direct bonding connections among dissimilar materials is even more dramatic in the case of HNCs incorporating all-magnetic compounds. In such cases, operation of exchange-coupling mechanisms and/or induction of extra magnetic anisotropy at the relevant heterointerfaces can transcribe into anomalous temperature dependence of the magnetization and unusually modified hysteretic behavior. ${ }^{1-4,25-27}$

Presently, full realization of the technological perspectives of HNCs remains yet hindered by the limited degree of synthetic development and property control with which heterostructures can be engineered. These issues are especially critical for associations of nonhomologous and/or structurally uncorrelated materials, for which the search for multifunctionality frequently conflicts with severe degradation of the native properties of any of the concerned material components due, for example, to unfavorable changes in electronic structure and/or to formation of defective interfaces. ${ }^{1-4,17,22-24,28-30}$ For such heterostructures, it also remains difficult to decouple mere proximity effects from unambiguous emergence of new or abnormal chemicalphysical phenomena. $^{1-4,18,23,24,29-40}$

One particular HNC class that has been tackled with limited success is represented by nanoheterostructures based on fluorescent semiconductors and magnetic materials, which are extremely attractive for environmental and biomedical applications, in the form of magnetically recoverable photocatalysts, on one side, and as tools for analytical separations, drug delivery, multimodal diagnostics and therapeutics, on the other side.

(14) Cheon, J.; Lee, J.-H. Acc. Chem. Res. 2008, 41, 1630-1640.

(15) Jun, Y.-w.; Choi, J.-s.; Cheon, J. Chem. Commun. 2007, 1203-1214.

(16) Choi, J. s.; Jun, Y. w.; Yeon, S. I.; Kim, H. C.; Shin, J. S.; Cheon, J. J. Am. Chem. Soc. 2006, 128, 15982-15983.

(17) Mokari, T.; Rothenberg, E.; Popov, I.; Costi, R.; Banin, U. Science 2004, 304, 1787-1790.

(18) Kudera, S.; Carbone, L.; Casula, M. F.; Cingolani, R.; Falqui, A.; Snoeck, E.; Parak, W. J.; Manna, L. Nano Lett. 2005, 5, 445-449.

(19) Dukovic, G.; Merkle, M. G.; Nelson, J. H.; Hughes, S. M.; Alivisatos, A. P. Adv. Mater. 2008, 20, 4306-4311.

(20) Costi, R.; Saunders, A. E.; Elmalem, E.; Salant, A.; Banin, U. Nano Lett. 2008, 8, 637-641.

(21) Lee, J.-S.; Shevchenko, E. V.; Talapin, D. V. J. Am. Chem. Soc. 2008 130, 9673-9675.

(22) (a) Saunders, A. E.; Popov, I.; Banin, U. J. Phys. Chem. B 2006, 110, 25421-25429. (b) Menagen, G.; Mocatta, D.; Salant, A.; Popov, I.; Dorfs, D.; Banin, U. Chem. Mater. 2008, 20, 6900-6902.

(23) Maynadiè, J.; Salant, A.; Falqui, A.; Respaud, M.; Shaviv, E.; Banin, U.; Soulantica, K.; Chaudret, B. Angew. Chem., Int. Ed. 2009, 48, 1814-1817.

(24) Kim, H.; Achermann, M.; Balet, L. P.; Hollingsworth, J. A.; Klimov, V. I. J. Am. Chem. Soc. 2005, 127, 544-546.

(25) Berkowitz, A. E.; Rodriguez, G. F.; Hong, J. I.; An, K.; Hyeon, T.; Agarwal, N.; Smith, D. J.; Fullerton, E. E. J. Phys. D: Appl. Phys. 2008, 41, 134007.

(26) Figuerola, A.; Fiore, A.; Di Corato, R.; Falqui, A.; Giannini, C.; Micotti, E.; Lascialfari, A.; Corti, M.; Cingolani, R.; Pellegrino, T.; Cozzoli, P. D.; Manna, L. J. Am. Chem. Soc. 2008, 130, 1477-1487.

(27) Casavola, M.; Falqui, A.; Garcia, M. A.; Garcia-Hernandez, M.; Giannini, C.; Cingolani, R.; Cozzoli, P. D. Nano Lett. 2009, 9, 366376.

(28) Casavola, M.; Grillo, V.; Carlino, E.; Giannini, C.; Gozzo, F.; Fernandez Pinel, E.; Garcia, M. A.; Manna, L.; Cingolani, R.; Cozzoli, P. D. Nano Lett. 2007, 7, 1386-1395.
Luminescent-magnetic HNCs also hold promise as miniaturized sources of spin-polarized current and/or light for implementation of future spintronic nanodevices. ${ }^{2}$ Available examples include Co@CdSe core@ shell nanospheres, ${ }^{24} \mathrm{FePt}-\mathrm{CdX}$ (where $\mathrm{X}=$ $\mathrm{S}, \mathrm{Se}$ ), ${ }^{29,30,34,40} \gamma-\mathrm{Fe}_{2} \mathrm{O}_{3}-\mathrm{MeX}$ (where $\mathrm{Me}=\mathrm{Cd}, \mathrm{Zn}, \mathrm{Hg}$ and $\mathrm{X}$ $=\mathrm{S}, \mathrm{Se}),{ }^{36-38}$ and $\mathrm{Fe}_{3} \mathrm{O}_{4}-\mathrm{Au}-\mathrm{PbX}^{39}$ hetero-oligomers, $\mathrm{CdS}$ and $\mathrm{CdSe}$ nanorods decorated with $\mathrm{PtNi} / \mathrm{PtCo}^{32}$ or Co domains, ${ }^{23}$ respectively. With a few exceptions, ${ }^{23,24}$ these prototypes generally suffer from almost complete luminescence abatement as well as from low thermal stability of the magnetization.

Here we report a colloidal two-step seeded-growth technique for the selective synthesis of three-component magnetic/ fluorescent HNCs with a matchstick-like topology with tunable geometric parameters. The newly developed HNCs individually comprise one metallic Co head connected to either apexes of a rod-shaped semiconductor section made of CdS embedding a spherical CdSe core. The specific heterostructure configuration achieved has been rationalized as resulting from the peculiar anisotropy reactivity of the noncentrosymmetric CdSe@CdS core@shell nanorods that have been employed to seed heterogeneous nucleation of Co from an organometallic precursor in a surfactant-free noncoordinating environment. These Co-tipped CdSe@CdS heterostructures show appreciable fluorescence despite occurrence of photoexcited electrons transfer from the semiconductor to the metal, and exhibit unusual ferromagneticlike behavior at room temperature, not otherwise achievable for corresponding physical mixtures made of unbound CdSe@CdS nanorods and Co nanocrystals.

\section{Experimental Section}

2.1. Synthesis Procedures. 2.1.1. Chemicals. All chemicals were of the highest purity available, anhydrous and used as received. Cadmium oxide (CdO, 99.5\%), 1-octadecene (ODE, 90\%) and oleic acid (OLAC, 90\%) were purchased from Sigma-Aldrich. Dicobalt octacarbonyl $\left(\mathrm{Co}_{2}(\mathrm{CO})_{8}\right.$ stabilized with $1-5 \%$ of hexane $)$, tri- $n$ octylphosphine oxide (TOPO 99\%), tri- $n$-octylphosphine (TOP, 97\%), elemental sulfur (S, 99\%), and elemental selenium (Se, $99,99 \%$ ) were purchased from Strem Chemicals. 1-Octadecylphosphonic acid (ODPA, 99\%) and 1-hexylphosphonic acid (HPA, 99\%) were purchased from Polycarbon Industries.

(29) Zanella, M.; Falqui, A.; Kudera, S.; Manna, L.; Casula, M. F.; Parak, W. J. J. Mater. Chem. 2008, 18, 4311-4317.

(30) He, S.; Zhang, H.; Delikanli, S.; Qin, Y.; Swihart, M. T.; Zeng, H. J. Phys. Chem C 2009, 113, 87-90.

(31) (a) Buonsanti, R.; Grillo, V.; Carlino, E.; Giannini, C.; Curri, M. L.; Innocenti, C.; Sangregorio, C.; Achterhold, K.; Parak, F. G.; Agostiano, A.; Cozzoli, P. D. J. Am. Chem. Soc. 2006, 128, 16953-16970. (b) Buonsanti, R.; Snoeck, E.; Giannini, C.; Gozzo, F.; Garcia-Hernandez, M.; Garcia, M. A.; Cingolani, R.; Cozzoli, P. D. Phys. Chem. Chem. Phys. 2009, 11, 3680-3691.

(32) Habas, S. E.; Yang, P.; Mokari, T. J. Am. Chem. Soc. 2008, 130, 3294 3295.

(33) Wetz, F.; Soulantica, K.; Falqui, A.; Respaud, M.; Snoeck, E.; Chaudret, B. Angew. Chem., Int. Ed. 2007, 46, 7079-7081.

(34) Gao, J.; Zhang, B.; Gao, Y.; Pan, Y.; Zhang, X.; Xu, B. J. Am. Chem. Soc. 2007, 129, 11928-11935.

(35) Pazos-Perez, N.; Gao, Y.; Hilgendorff, M.; Irsen, S.; Perez-Juste, J.; Spasova, M.; Farle, M.; Liz-Marzan, L. M.; Giersig, M. Chem. Mater. 2007, 19, 4415-4422.

(36) Selvan, S. T.; Patra, P. K.; Ang, C. Y.; Ying, J. Y. Angew. Chem., Int. Ed. 2007, 46, 2448-2452.

(37) Kwon, K. W.; Shim, M. J. Am. Chem. Soc. 2005, 127, 10269-10275.

(38) McDaniel, H.; Shim, M. ACS Nano 2009, 3, 434-440.

(39) Shi, W.; Zeng, H.; Sahoo, Y.; Ohulchanskyy, T. Y.; Ding, Y.; Wang, Z. L.; Swihart, M.; Prasad, P. N. Nano Lett. 2006, 6, 875-881.

(40) Gu, H. W.; Zheng, R. K.; Zhang, X. X.; Xu, B. J. Am. Chem. Soc. 2004, 126, 5664-5665. 
2.1.2. Synthesis of Spherical CdSe Nanocrystals. To synthesize $\mathrm{CdSe}$ nanocrystals (average diameter $=3.5 \mathrm{~nm}$ ) to be used as seeds for the growth of CdSe@CdS core@ shell nanorods, TOPO (3.00 $\mathrm{g})$, ODPA (0.28 g) and $\mathrm{CdO}(0.06 \mathrm{~g})$ were placed into a threenecked round-bottom flask and degassed at $150{ }^{\circ} \mathrm{C}$ for $1 \mathrm{~h}$. The reaction mixture was then heated to $370{ }^{\circ} \mathrm{C}$ under $\mathrm{N}_{2}$ atmosphere, during which the initially reddish solution became colorless, indicating the formation of a Cd-ODPA complex. At this point $1.5 \mathrm{~g}$ of TOP was added to the flask. Subsequently, a TOP/Se solution (58 mg of elemental Se in $360 \mathrm{mg}$ of TOP) was injected quickly (in less than $2 \mathrm{~s}$ ) at $370{ }^{\circ} \mathrm{C}$ and the reaction mixture was annealed at this temperature for an additional $30 \mathrm{~s}$, after which it was allowed to cool down upon removal of the heating mantle. The formed $\mathrm{CdSe}$ nanocrystals were precipitated by addition of methanol, then washed, and finally redispersed in TOP for further use. To synthesize smaller CdSe nanocrystals $(\sim 2.5 \mathrm{~nm})$, the reaction was stopped immediately after the injection of the TOP/Se solution.

2.1.3. Synthesis of CdSe@CdS Core@Shell Nanorods (NRs). In a typical synthesis of CdSe@CdS core@shell NRs with aspect ratio of $\sim 5,{ }^{6} \mathrm{CdO}(0.06 \mathrm{~g})$ was mixed together with TOPO (3.00 g), ODPA (0.290 g) and HPA (0.08 g) in a flask. After pumping the flask to vacuum for about $1 \mathrm{~h}$ at $150{ }^{\circ} \mathrm{C}$, the resulting solution was heated to $350{ }^{\circ} \mathrm{C}$ under $\mathrm{N}_{2}$ atmosphere. At about $300{ }^{\circ} \mathrm{C}$, the reddish $\mathrm{CdO}$ powder dissolved, generating a colorless, transparent solution. At this point $1.5 \mathrm{~g}$ of TOP was added. After a temperature of about $350{ }^{\circ} \mathrm{C}$ was recovered, a solution made by mixing $\mathrm{S}(0.120$ $\mathrm{g})$, TOP $(1.50 \mathrm{~g})$, and $412 \mu \mathrm{M} \mathrm{CdSe} / \mathrm{TOP}$ solution $(206 \mu \mathrm{L}$ containing $3.5-\mathrm{nm}$ CdSe nanocrystals) was rapidly injected into the flask. The reaction was allowed to proceed for $6 \mathrm{~min}$, after which the mixture was cooled to $60{ }^{\circ} \mathrm{C}$ and halted by adding anhydrous toluene. The flask was then transferred to the glovebox and the formed CdSe@CdS NRs were precipitated upon addition of anhydrous methanol. After repeated washing with methanol, the NRs were dispersed in toluene. On the other hand, to synthesize longer NRs (with aspect ratio of $\sim 20-25$ ), the amounts of $\mathrm{CdO}$ and $\mathrm{S}$ used in the synthesis were changed to 0.09 and $0.06 \mathrm{~g}$, respectively, while the other parameters were kept constant. ${ }^{6}$

2.1.4. Synthesis of CdSe@CdS-Co Hybrid Nanocrystals (HNCs). In a typical synthesis, $5 \mathrm{~mL}$ of ODE was first loaded into a $50 \mathrm{~mL}$ three-neck flask and pumped to vacuum for $1 \mathrm{~h}$ at $150{ }^{\circ} \mathrm{C}$. Then the flask was cooled down to $80{ }^{\circ} \mathrm{C}$ under $\mathrm{N}_{2}$ and a calibrated amount of CdSe@CdS NRs in toluene was added. The resulting mixture was again pumped to vacuum for another $30 \mathrm{~min}$ to remove toluene and oxygen residuals. The concentration of the NRs inside the reaction flask was approximately equal to $1.5 \times 10^{-9} \mathrm{M}$. To grow the HNCs, the NR-loaded solution was heated to 200-250 ${ }^{\circ} \mathrm{C}$ under $\mathrm{N}_{2}$ and allowed to equilibrate for $15 \mathrm{~min}$, after which a $2-2.5 \mathrm{~mL}$ of a degassed $\mathrm{Co}_{2}(\mathrm{CO})_{8} / \mathrm{ODE}$ solution $(0.05 \mathrm{M})$ was injected to the vigorously stirred mixture at a rate of $0.05 \mathrm{~mL} / \mathrm{min}$. The color of the solution slowly changed from yellow to black, indicating Co nucleation. The $\mathrm{Co}_{2}(\mathrm{CO})_{8}$ decomposition was allowed to proceed to completion for an additional $15 \mathrm{~min}$, following which $0.1 \mathrm{~mL}$ of previously degassed OLAC was added to the flask to stabilize the HNCs. The reaction was eventually annealed for an additional 5-15 $\mathrm{min}$, and then halted upon removing the heating source.

Once cooled to room temperature, the crude reaction mixture was mixed with $5 \mathrm{~mL}$ of anhydrous 2-propanol in a glovebox, which led to precipitation of the nanocrystal products. The HNCs were separated from unreacted NRs by application of a $1 \mathrm{~T}$ bar magnet to one side of a vial containing a nanocrystal dispersion in 2-propanol. The solution containing unreacted NRs was then decanted. Finally the collected HNCs resulted in being dispersible in variety of nonpolar solvents, such as toluene, chloroform, and hexane.

2.1.5. Synthesis of Co Nanocrystals. Cobalt nanocrystals were independently prepared using the same procedure adopted for HNC synthesis, but in the absence of preformed CdSe@CdS NR seeds in the reaction flask. To increase the Co nanocrystal size, proportionally larger amounts of $\mathrm{Co}_{2}(\mathrm{CO})_{8} / \mathrm{ODE}$ precursor solution were injected into the vessel. After the synthesis, the nanocrystals were extracted and purified, as described above, and used to prepare appropriate CdSe@CdS NR/Co physical mixtures that served for comparative evaluation of the optical and magnetic properties of the HNC samples.

2.2. Characterization. 2.2.1. Transmission Electron Microscopy (TEM). Low-resolution TEM images were recorded on a JEOL JEM 1011 microscope operating at $100 \mathrm{kV}$. Phase-contrast highresolution TEM (HRTEM) measurements were performed with a JEOL $2100 \mathrm{~F}$ microscope, equipped with a field emission gun working at an accelerating voltage of $200 \mathrm{kV}$. Energy-filtered TEM (EFTEM) experiments were performed on a JEOL JEM-2200FS microscope equipped with an in-column Omega filter. The spherical aberration was corrected to less than $5 \mu \mathrm{m}$. A contrast aperture was used in order to reduce the chromatic aberration and achieve a spatial resolution in the filtered images of far less than $1 \mathrm{~nm}$ (as expected on the basis of delocalization of inelastic scattering). ${ }^{56}$ The elemental maps were obtained by using the conventional threewindow method. ${ }^{57}$ The samples for TEM analyses were prepared in a glovebox by depositing a few drops of a dilute HNC solution onto carbon-coated $\mathrm{Cu}$ grids that were immediately transferred to the microscope.

2.2.2. Powder X-ray Diffraction (XRD). XRD measurements were performed with a Rigaku-Inel diffractometer equipped with a $12 \mathrm{~kW}$ ceramic tube with a molybdenum anode, a Ge(111) singlecrystal monochromator, and a CPS120 INEL detector. For the measurements, dry nanocrystal powders, homogeneously loaded into Lindemann capillaries $(0.5 \mathrm{~mm}$ in diameter $)$ under the inert atmosphere of a glovebox, were measured in reflection geometry. Data were collected at a fixed incident angle of about 1 degree. Note that these XRD measurements could not allow crystal shape anisotropy to be appreciated from inhomogeneous peak broadening due to intrinsic limitations of the instrumental resolution function that prohibited unambiguous discrimination of crystalline domains larger than $\sim 4 \mathrm{~nm}$. Under comparable measuring conditions, the XRD scattered intensities for $\mathrm{CdSe} @ \mathrm{CdS}$ nanorods were $\sim 10$ times larger than those found for pure Co nanocrystals.

2.2.3. Elemental Analysis. The $\mathrm{Cd}$ and $\mathrm{Co}$ atomic element content of the samples was measured via Inductively Coupled Plasma Atomic Emission Spectroscopy (ICP-AES), using a Varian Vista $\mathrm{AX}$ spectrometer. Samples were digested either in $\mathrm{HNO}_{3}$ or in $\mathrm{HCl} / \mathrm{HNO}_{3} 3: 1(\mathrm{v} / \mathrm{v})$.

2.2.4. Magnetic Measurements. The magnetic properties of the samples were investigated by SQUID magnetometry, normalizing the results to the actual Co mass in the samples, as determined by ICP-AES measurements. Measurements of static magnetization and hysteretic behavior were performed with a Quantum Design MPMS SQUID magnetometer, equipped with a superconducting magnet that could generate fields as large as $5 \mathrm{~T}$. Zero-field-cooled (ZFC) magnetization curves were measured by cooling samples in a zero magnetic field and then by increasing the temperature in an applied field of $2.5 \mathrm{mT}$. Field-cooled (FC) magnetization curves were recorded by cooling the samples in the same field of $2.5 \mathrm{mT}$. The field dependence of the magnetization (hysteresis loop) was recorded up to $\pm 5 \mathrm{~T}$ at $T=2.5$ and $310 \mathrm{~K}$. The saturation magnetization $\left(M_{\mathrm{S}}\right)$ values were derived from corresponding plots of $M$ versus $1 / \mathrm{H}$ by extrapolation of the $M$ values corresponding to $1 / \mathrm{H} \rightarrow 0$.

2.2.5. Steady-State UV-Vis Absorption and Photoluminescence (PL) Spectroscopy. Absorption measurements were carried out using a Varian Cary 300 UV-vis spectrophotometer. PL spectra were recorded with a Varian Cary Eclipse fluorescence spectrophotometer with an intense Xenon flash lamp. The photoluminescence QY of the various samples was estimated by the "gradient method", using Rhodamine G6 as reference fluorescent dye, and exciting all the samples at $488 \mathrm{~nm}$. This method has been discussed extensively elsewhere. ${ }^{58}$ 
2.2.6. Time-Resolved Photoluminescence (TRPL). TRPL measurements were performed at room temperature. The nanocrystals were excited with the second harmonic $(405 \mathrm{~nm})$ of a Ti:sapphire laser (with $80 \mathrm{fs}$ pulse duration and $80 \mathrm{MHz}$ repetition rate). The PL signal was collected by a spectrograph and detected by a streak camera (temporal resolution of $12 \mathrm{ps)}$ ). To avoid spectral effects due to nanocrystal orientation, energy transfer among the nanocrystals, and/or photodegradation, all of the measurements were carried out using nanocrystals dissolved in toluene at micromolar concentration. In addition, the excitation density was kept low to avoid multicarrier effects.

\section{Results and Discussion}

3.1. Size-Morphological Characterization of Synthesis Products. Our synthetic approach relies on two sequential seededgrowth steps, as sketched in Scheme 1. In the first step (Scheme 1, part a),CdSe@CdS core@shell nanorods (NRs) are synthesized by rapidly injecting a room-temperature tri-n-octyl phosphine sulphide (TOP/S) solution and preformed monodisperse $\mathrm{CdSe}$ nanocrystal seeds into a hot bath of Cd-surfactant complexes obtained by decomposing $\mathrm{CdO}$ in a TOPO/ODPA/ HPA mixture at $350-380{ }^{\circ} \mathrm{C}$. ${ }^{6}$ This technique guarantees preferential heterogeneous nucleation and anisotropic growth of $c$-axis elongated wurtzite $\mathrm{CdS}$ onto the primary $\mathrm{CdSe}$ seeds, ultimately resulting in CdSe@CdS core@shell NRs that individually embody one of the original CdSe nanocrystals in an eccentric position across the CdS section, that is, in a region between $1 / 3$ and $1 / 4$ of their overall longitudinal length (Scheme 1 , part a), as previously verified by geometric phase analysis of aberration-corrected HRTEM images. ${ }^{6}$ Unlike their CdSeor CdS-only counterparts, ${ }^{8,17,23,32,41}$ such heterostructured NRs represent convenient starting substrates for the synthesis of fluorescent HNCs equipped with a magnetic metal for two main reasons. First, they can be grown with uniform and tunable geometric parameters over a wide dimensional range by simply adjusting the $\mathrm{CdSe}$ seed size and/or the relative $\mathrm{CdSe}$ to $\mathrm{CdO} / \mathrm{S}$ precursor proportions in the synthesis. Second, due to their crystal structure, shape anisotropy, and type-I band alignment, ${ }^{42}$ they offer a rich selection of optical properties, such as large absorption cross-section, linearly polarized emission, and wavelength-tunable fluorescence with quantum yields ranging from 70 to $10 \%$, depending on the NR aspect ratio. ${ }^{6}$ As photoluminescence originates from band-edge carrier recombination at the inner CdS-buried CdSe core, and nonradiative exciton decay channels (e.g., trapping/detrapping processes at the surface) are negligible, such NRs can be expected to better withstand fluorescence quenching that may occur upon formation of metalsemiconductor heterojunctions. ${ }^{1,4,17,22,24,29,30,34,40}$

In a second step (Scheme 1, part b), purified CdSe@CdS NRs $\left(1.5 \times 10^{-9} \mathrm{M}\right)$ are used themselves as substrate seeds for accommodating a secondary Co component in noncoordinating ODE heated at $200-240{ }^{\circ} \mathrm{C}$ under inert atmosphere, into which appropriate amounts of an organometallic $\mathrm{Co}_{2}(\mathrm{CO})_{8}$ precursor solution $(0.05 \mathrm{M})$ are injected at a slow rate $(0.05 \mathrm{~mL} / \mathrm{min})$ controlled by a syringe pump. Note that in this stage, no extra surfactants are introduced into the NR-loaded ODE environment in the flask. After reaction completion (Scheme 1, part c), excess OLAC is added to the growing mixture to stabilize the as-formed $\mathrm{HNCs}$ and prevent them from aggregating via coalescence of

(41) Mokari, T.; Sztrum, C. G.; Salant, A.; Rabani, E.; Banin, U. Nat. Mater. $\mathbf{2 0 0 5}, 4,855$.

(42) Steiner, D.; Dorfs, D.; Banin, U.; Della Sala, F.; Manna, L.; Millo, O. Nano Lett. 2008, 8, 2954-2958.
Scheme 1. Sketch of the Synthetic Procedure Devised to Synthesize the $\mathrm{HNCs}^{a}$

\section{CdSe seeds}
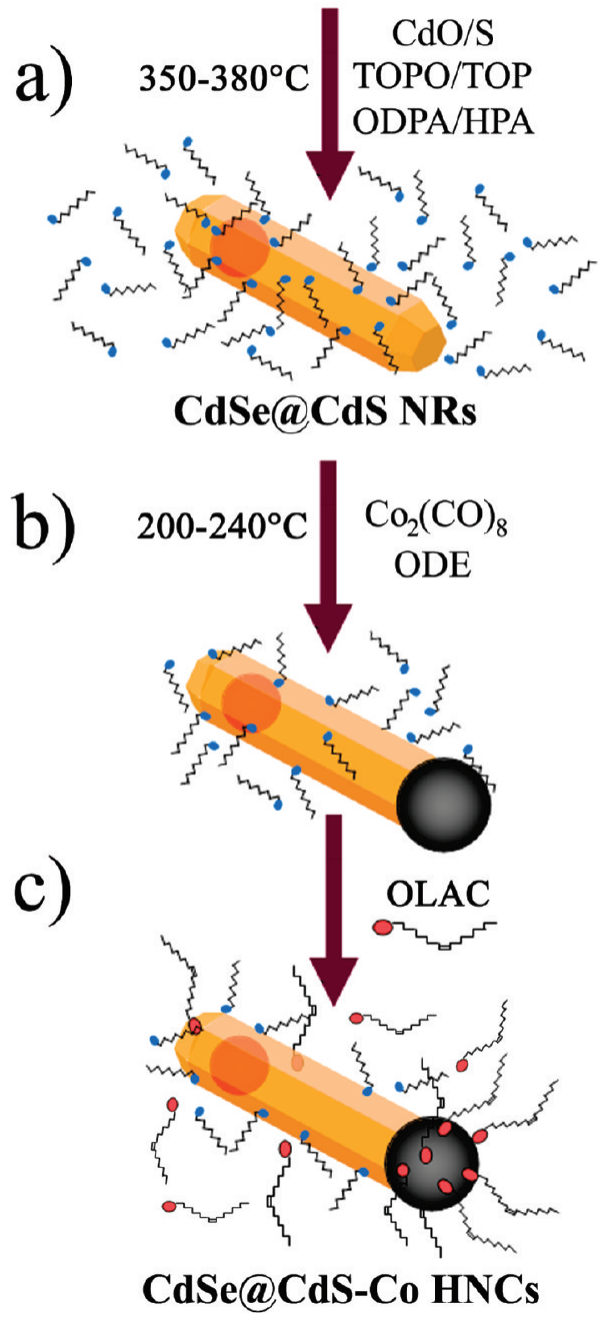

a (a) Preparation of noncentrosymmetric CdSe@CdS NRs from CdSe spherical nanocrystals in a quaternary surfactant mixture (TOPO/TOP/ ODPA/HPA) at $350-380^{\circ} \mathrm{C}$; (b) thermal decomposition of $\mathrm{Co}_{2}(\mathrm{CO})_{8}$ in the presence of previously purified NRs in non-coordinating ODE at $200-240^{\circ} \mathrm{C}$ (note that in this stage, the only surfactant species present in the reaction environment are those desorbing from the NRs); (c) stabilization of the as-derived heterostructures after Co growth completion by addition of excess OLAC.

their metal tips. The product is extracted by conventional alcohol-induced flocculation and centrifugation, and thoroughly purified by repeated redispersion/precipitation cycles. When required, the HNCs can be eventually size-sorted from unreacted NRs by combining alcohol destabilization and magnet-assisted recovery, which confirms that the as-derived heterostructures, being indeed made of permanently joint CdSe@CdS NR and Co sections, are the highest-mass objects in the particle population, and are magnetically responsive. Finally, the hydrophobically capped heterostructures can be fully dissolved in a variety of nonpolar solvents (e.g., chloroform, toluene, hexane).

The representative low-magnification TEM galleries in Figure 1 demonstrate the degree of topological selectivity achievable in typical syntheses seeded with regularly sized and shaped 


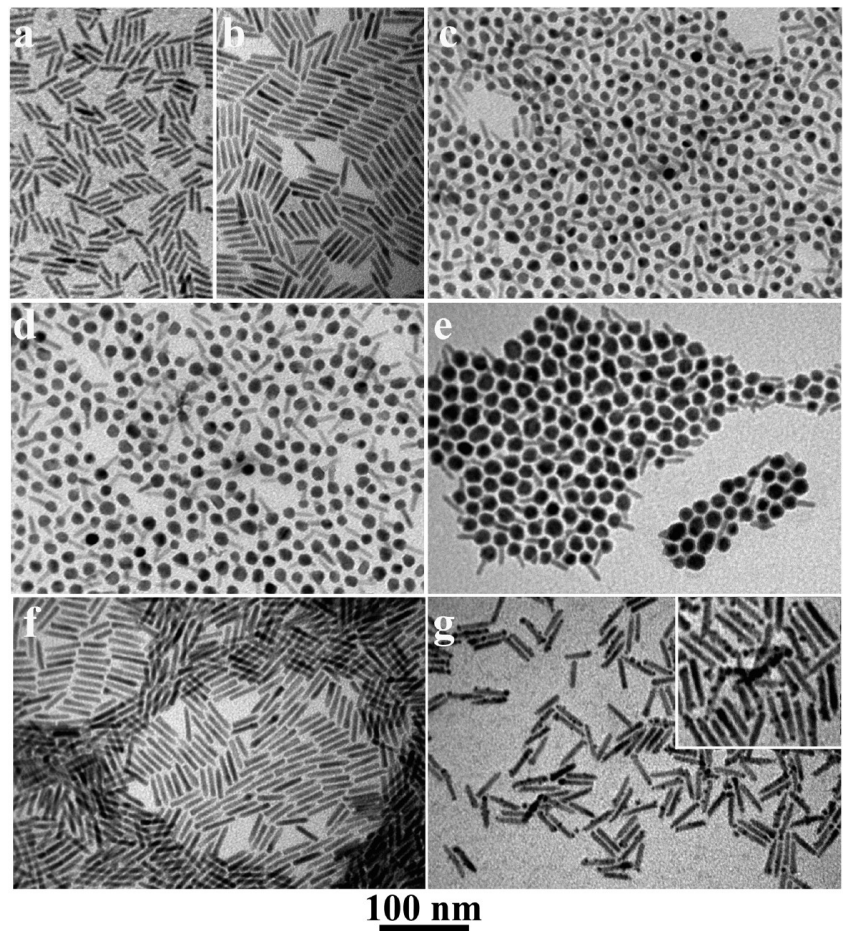

Figure 1. Representative low-magnification TEM overview of: $(a-b)$ CdSe@CdS core@shell NRs with different aspect ratios, obtained by seeding CdS growth with spherical CdSe nanocrystals. (c-e) Matchsticklike HNCs made of Co-tipped CdSe@CdS NRs with different geometric parameters, for which the Co head size varies from $\sim 4$ to $\sim 15 \mathrm{~nm}$, respectively. (f) CdS-only NRs obtained by a seeded-growth approach starting from spherical CdS nanocrystals; (g) HNCs made of Co-decorated CdS-only NRs (a magnified image of the sample is shown in the inset).

CdSe@CdS NRs with different geometric parameters. The seed population generally contains both regularly rod-like and pencilshaped NRs in approximately equal proportions (Figure 1a-b). Tendency toward one-sided NR sharpening arises from the dissimilar development rates of the wurtzite lattice along the $\langle 001\rangle$ and the $\langle 00 \overline{1}\rangle$ directions under kinetically overdriven growth conditions. ${ }^{1-4,6}$ Upon reaction with the $\mathrm{Co}_{2}(\mathrm{CO})_{8}$ precursor, the formation of $\mathrm{Co}-\mathrm{CdSe} @ \mathrm{CdS} \mathrm{HNCs}$ consisting of Co-tipped NRs is supported by the observation of matchsticklike objects exhibiting a distinguished image contrast variation across their profile (Figure 1c-e). On the basis of the comparatively higher electron density of Co relative to $\mathrm{CdS}$, the roughly spherical head attached to either termination should be ascribed to metallic Co, while the tail section of each heterostructure can be interpreted as incorporating one of the original CdSe@CdS NR seeds, albeit with 5\%/10\% reduced diameter/length. These attributions are consistent with the known decomposition paths of $\mathrm{Co}_{2}(\mathrm{CO})_{8}$ under inert atmosphere, ${ }^{43-48}$ and with pertinent control experiments that confirm occurrence of little CdSe@CdS NR size shrinking upon prolonged heating in hot ODE (i.e., in the absence of extra stabilizers and/or added $\mathrm{Cd} / \mathrm{S}$ molecular precursors). Under optimized conditions, the slow injection of gradually larger $\mathrm{Co}_{2}(\mathrm{CO})_{8}$ amounts to the seedcontaining flask allows the Co dimensions to be progressively tuned from about $\sim 3-4 \mathrm{~nm}$ up to $\sim 14 \mathrm{~nm}$, with typical size variances of the order of $10-15 \%$ (Figure $1 \mathrm{c}-\mathrm{e}$ ). The HNC yield (expressed in terms of fraction of seeds that have reacted with the cobalt precursor and converted to CdSe@CdS-Co heterostructures) typically oscillates in between $50-70 \%$. On the other hand, the formation of separate Co nanocrystals, which proceeds at the expense of HNC yield, is observed only at remarkably higher reaction temperatures $\left(>245^{\circ} \mathrm{C}\right)$, upon fast precursor injection, and/or in the presence of deliberately added extra surfactants in the reaction medium (Figure S1 in the Supporting Information). Additionally it deserves reporting that the specific one-tipped Co growth mode is retained for CdSe@CdS NRs with aspect ratios of less than 10, beyond which a substantial fraction of binary heterostructures with Co domains accommodated on the longitudinal sidewalls of their rod-like tail is observed (Figure S2 in the Supporting Information). Even more interestingly, a nonselective topological regime distinguishes Co growth performed on CdS-only NRs of comparable size and shape homogeneity (synthesized by a CdSseeded-growth approach similar to that used for preparing the CdSe@CdS NRs ${ }^{6}$ ), regardless of their dimensions (Figure $1 \mathrm{f}-\mathrm{g}$ ). These findings preliminarily suggest that not only the geometric parameters but also the inner core@shell structure of the seeds should come into play in dictating the HNC architectures ultimately achievable.

3.2. Structural-Compositional Studies on the HNCs. The structural-compositional details of the heterostructures have been studied by combined HRTEM and powder XRD analyses. Representative results are illustrated in Figure 2 and Figure 3, respectively.

Phase-contrast HRTEM investigations of HNCs captured under different zone axes (Figure 2a, d, f), along with the twodimensional fast Fourier transform (2D-FFT) of the relevant images (Figure $2 b-c, e, g$, respectively) reveal that the heterostructures indeed retain $c$-axis elongated wurtzite $\mathrm{CdS}$ into their rod-shaped tail section, while the spherical portions at the tips are indentified to be single-crystalline metallic Co domains in the hexagonal close-packed (hcp) structure in most cases (note that the low statistically informative value of HRTEM analyses does not allow the presence of cubic $\varepsilon$-Co phase in the samples to be ruled out completely). As preliminarily suggested by the low-magnification TEM overview (Figure 1), the HRTEM inspection indeed clarifies that the Co domains are always accommodated on the basal sides of NRs, whereby the largest interfacial area that Co shares with CdS seeds spans the seed diameter (i.e., short axis size). Distinctive set of fringes can be unambiguously identified, such as those corresponding to the (002) and (110) planes of bulk CdS $\left(d_{002}{ }^{\mathrm{CdS}}=0.34 \mathrm{~nm}\right.$ and $\left.d_{110}{ }^{\mathrm{CdS}}=0.20 \mathrm{~nm}\right)$, and the (002) and (101) planes of bulk hcpCo $\left(d_{002}{ }^{\text {hcp-Co }}=0.20 \mathrm{~nm}, d_{101}{ }^{\text {hcp-Co }}=0.19 \mathrm{~nm}\right)$. In most cases, the particular orientations under which the two lattices are captured do not permit any site-coincidence crystallographic relationships between them to be assessed. In addition, the angles between the most frequently encountered families of lattice planes in the respective material domains do not exhibit recurring values in distinct heterostructures, which points to attainment of nonepitaxial metal-semiconductor junctions. ${ }^{17,19,22,32}$

(43) Puntes, V. F.; Zanchet, D.; Erdonmez, C. K.; Alivisatos, A. P. J. Am. Chem. Soc. 2002, 124, 12874-12880.

(44) Park, J.-I.; Kang, N.-J.; Jun, Y.-W.; Oh, S. J.; Ri, H.-C.; Cheon, J. Chem. Phys. Chem. 2002, 3, 543-547.

(45) Tracy, J. B.; Weiss, D. N.; Dinega, D. P.; Bawendi, M. G. Phys. Rev. $B$ 2005, 72, 064404.

(46) Yang, H. T.; Shen, C. M.; Su, Y. K.; Yang, T. Z.; Gao, H. J.; Wang, Y. G. Appl. Phys. Lett. 2003, 82 (26), 4729-4731.

(47) Cheng, G.; Dennis, C. L.; Shull, R. D.; Walker, A. R. H. Langmuir 2007, 23, 11740-11746.

(48) de Silva, R. M.; Palshin, V.; de Silva, K. M. N.; Henry, L. L.; Kumar, C. S. S. R. J. Mater. Chemistry 2008, 18, 738-747.

(49) Morello, G.; Della Sala, F.; Carbone, L.; Manna, L.; Maruccio, G.; Cingolani, R.; De Giorgi, M. Phys. Rev. B 2008, 78, 195313.

(50) Koshin, H.; Takeshi, T.; Shinpei, Y.; Kazunari, M.; Yoshihiko, K. Phys. Rev. Lett. 2008, 100, 207404. 


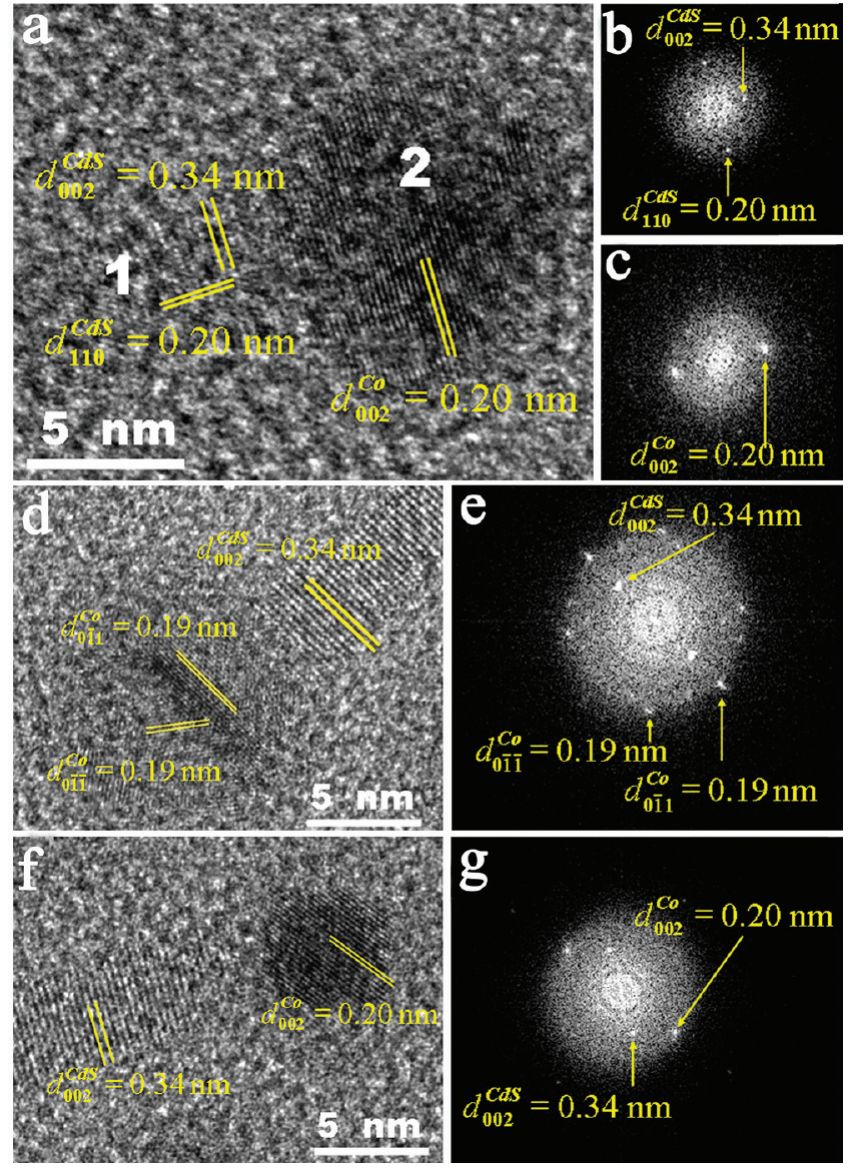

Figure 2. HRTEM characterization of a representative sample of HNCs (made of a $\sim 5 \times \sim 20 \mathrm{~nm}$ CdSe $@$ CdS tail section and $\sim 8 \mathrm{~nm}$ Co head): (a, $d, f)$ phase-contrast HRTEM images of individual HNCs observed under different orientations. (b, c) Two-dimensional fast fourier transform (2DFFT) patterns calculated from the wurtzite CdS rod section (marked as "1" in panel a) in its $\langle 1,1,0\rangle$ zone axis and from the spherical $h c p$-Co head (marked as " 2 " in panel b), respectively. (e) FFT pattern obtained from the entire HNC seen in panel d; (g) 2D-FFT pattern obtained from the HNC in panel f.

However, given the large differences in lattice parameters between the $\mathrm{Co}$ and $\mathrm{CdS}$ hexagonal crystal structures (by about $40 \%$ ), it is likely that the two lattices will experience significant interfacial strain at the relevant junction regions.

In agreement with the HRTEM data, the XRD profile of the initial CdSe@CdS NRs (trace a in Figure 3) can be closely indexed to the wurtzite (hexagonal) $\mathrm{CdS}$, as expected on the basis of the significantly larger abundance of the CdS shell mass relative to the CdSe core component. The NR crystal phase appears to be preserved in the CdSe@CdS-Co HNCs derived from such seeds (trace b in Figure 3). Although some minor differences are appreciable, no Co phases are clearly discernible due to various concurrent effects, including the strong reflection overlapping, the significant peak broadening, and the much lower X-ray scattering power of the minority metal component relative to wurtzite CdSe@CdS phase (see Experimental Section). Nevertheless, such modified features in the HNC pattern can arise not only from the presence of the Co components, but also from slight changes in the dimensions and/or in the degree of crystallinity of the NR section, which may occur during heterostructure growth. Such XRD contributions, combined with the effects produced by the nanocrystalline domain size distribution, are likely to overwhelm the signature of interfacial strain associated to the $\mathrm{CdS} / \mathrm{Co}$ heterojunctions, which

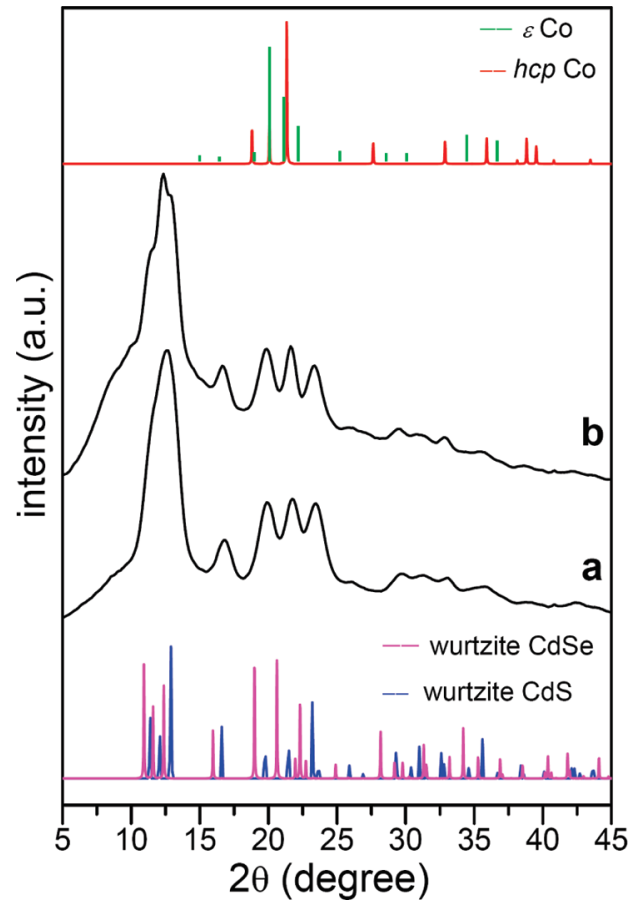

Figure 3. XRD characterization of a representative sample of HNCs (made of a $\sim 5 \times \sim 20 \mathrm{~nm} \mathrm{CdSe@CdS} \mathrm{tail} \mathrm{section} \mathrm{and} \sim 8 \mathrm{~nm}$ Co head): (a) XRD patterns of the starting NRs and (b) HNCs derived thereof, along with the reference patterns of bulk wurtzite CdSe, wurtzite CdS, hcp-Co and $\varepsilon$-Co (the cobalt weight percentage in the HNC sample is about 33\%, according to ICP-AES analysis).

can not therefore be decoupled unambiguously by Rietveldbased whole-profile fitting methods.

To gain more information regarding the relative positioning of the Co heads to the CdSe core in the NR section, the local chemical composition of the samples has been investigated by EFTEM. As a representative case of study, Figure 4 addresses a group of nanocrystals that were not subjected to any sizeselective separation. The elastic zero-loss EFTEM image (Figure 4a), obtained by filtering with an energy window of $10 \mathrm{eV}$ around the elastic peak, clearly shows the presence of both matchstick- and rod-like objects, corresponding to HNCs and isolated unreacted NRs, respectively. The image contrast is similar to that detectable with conventional medium-magnification TEM due to the dominance of elastic and Bragg scattering. Taking the zero-loss image as a reference, the corresponding inelastic EFTEM image generated by filtering electrons at the $\mathrm{S} \mathrm{L}_{2,3}$ edge (165 eV) represents the map of $\mathrm{S}$ species distribution across the same visible nanostructures (green color in Figure 4b). Unfortunately, complementary information regarding $\mathrm{Se}$ elements could not be acquired due to negligible signal intensity associated to the extremely small CdSe domains. The S map has been superimposed to an EFTEM image recorded in the energy loss region preceding the $\mathrm{S}_{2,3}$ edge, where the contrast is mainly due to Co $\mathrm{M}_{1}$ and $\mathrm{M}_{2,3}$ shells (blue color in Figure 4b), and to the elastic EFTEM image (red color in Figure 4b), the latter reflecting the supporting carbon film of the TEM grid. Although the overall signal arising from the $\mathrm{SL}_{2,3}$ is generally low, a careful inspection of the overall composite false-color chemical map in Figure 4 allows recognition of negative contrast regions across the NR sections, which can be ascribed to $\mathrm{S}$ signal reduction in correspondence of the inner $\mathrm{CdSe}$ cores. The CdSe domains therefore appear to be located asymmetrically along the CdS sections both of the bare NRs and the HNCs. In the 


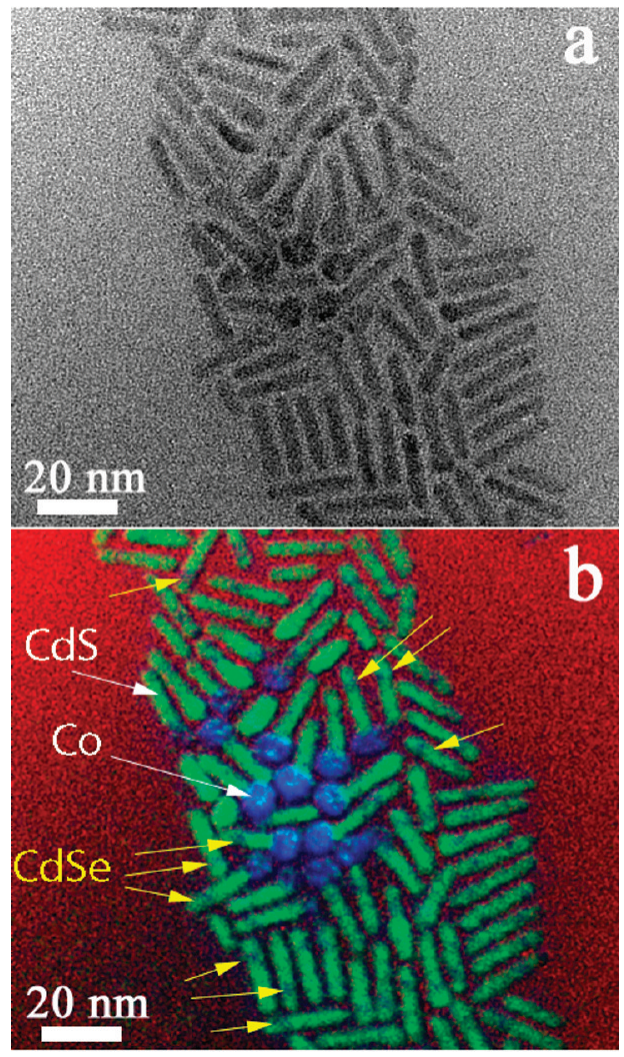

Figure 4. EFTEM investigation of NR and HNC samples. (a) Elastic zeroloss EFTEM image of a group of bare CdSe@CdS NRs and of matchsticklike Co-tipped CdSe@CdS NR heterostructures (b) Corresponding composite false-color chemical map obtained upon superimposing: an inelastic EFTEM image obtained by filtering at the S L2,3 edge (green); an inelastic EFTEM image recorded in the energy loss region close to the Co $M$ edges (blue); the elastic EFTEM image (red), the latter reflecting the supporting carbon film of the TEM grid. The yellow arrows indicate some examples of negative contrast regions across the nanorod sections, which can be associated to the presence of the buried CdSe cores.

latter, the CdSe regions are most frequently found in a position that is diametrically opposite to the apex bearing the Co tip.

3.3. Growth Mechanism of the HNCs. The mechanism through which the HNCs are achieved has been disclosed by time-dependent TEM monitoring of the nanostructure products along the synthesis course. Since the early reaction stages, tiny Co patches are detected on the apexes of the NRs, which enlarge progressively while the relative fraction of heterostructures within the overall particle population remains almost invariant over time (Figure S3 in the Supporting Information). This evolution discredits the occurrence of crystal-oriented-attachment pathways involving the directional fusion of homogeneously generated Co nanocrystals to the NR tips, which should instead lead to a progressive increase in the population of $\mathrm{HNCs}$ at the expense of other nanoparticle types, in contrast to experimental findings. On the other side, the almost exclusive formation of binary HNCs based on nanorod-sphere pairs further excludes any sort aggregation-based growth mechanism, which should in fact result in various types of NR- or sphere-based oligomers, and/or combinations of them. Accordingly, control experiments in which mixtures of $\mathrm{CdSe} @ \mathrm{CdS} \mathrm{NRs}$ and preformed Co nanocrystals (i.e., provided by a separate synthesis) are heated up for extended periods demonstrate a scarce probability to create HNCs by stimulating thermal coalescence.

On the basis of the above observations, it can be safely concluded that the HNCs originate through a short burst of heterogeneous Co nucleation onto the NR seeds, which is followed by selective autocatalytic growth of the initially deposited metal nuclei upon addition of reactive monomer species from the bulk solution. As a further indirect proof in support of this mechanism, it should be considered that the Co tips are attained in the $h c p$-structure, whereas pertinent control experiments indicate that free-standing Co nanocrystals synthesized under identical conditions in the absence of NRs are preferentially achieved in the cubic $\varepsilon$-phase (Figure S4 in the Supporting Information). ${ }^{28,43-48}$ This fact suggests that the particular NR seed facets onto which Co is deposited are likely to play a role as crystal-phase-directing substrates for the metal.

The result of preparing unique semiconductor/magnetic matchstick-like HNCs upon accomplishing Co growth in the presence of NRs that specifically possess both an eccentric CdSe@CdS core@shell configuration and suitable dimensional parameters demonstrates that our colloidal technique achieves proper control over the fundamental nucleation/growth processes underlying heterostructure formation, on one side, and guarantees topological specificity by use of suitably engineered nanocrystal seed substrates, on the other side.

The synthesis dynamics can be rationalized within the frame of the classical nucleation theory (CNT), according to which the activation energy required for $\mathrm{Co}$ to nucleate heterogeneously and grow on pre-existing nanocrystals seeds can be considerably lower than the barrier that has to be overcome for separate Co embryos to nucleate homogeneously in the bulk solution. ${ }^{4}$ This is practically realized by a synthetic scheme that sets the following favorable circumstances for heterostructure growth.

First, the freshly prepared CdSe@CdS NRs heated up in a hot ligand-free noncoordinating medium (ODE) can become highly susceptible toward Co deposition due to the pristine capping surfactants as well as the outermost exposed material layers being etched out of their surface so as to re-establish an equilibrium between the NRs and solution species (i.e., monomers, stabilizing agents) (Scheme 1, part b). ${ }^{27}$ This interpretation actually explains the slight size shrinking of the NR seeds, which has been observed to occur along the course of Co growth (cf. Figure 1 and Figure S3 in the Supporting Information). Additionally, in these conditions a reaction of $\mathrm{Co}_{2}(\mathrm{CO})_{8}$ with the highly reactive $\mathrm{Cd} / \mathrm{S}$-containing monomers released from the seeds to the solution could result in an initial nucleation of a thin $\mathrm{Co}_{x} \mathrm{~S}_{y}$ buffer layer ${ }^{59}$ onto the NRs, which could, in turn, favor subsequent $\mathrm{Co}$ deposition upon interfacial strain alleviation.

Second, a judiciously slow-rate delivery of the $\mathrm{Co}_{2}(\mathrm{CO})_{8}$ precursor to the seed solution kept at a suitable temperature prevents Co monomers from accumulating fast in the solution and reaching the critical supersaturation threshold for parasitic homogeneous nucleation to take place. Under these conditions, the metal monomers produced by precursor decomposition are therefore efficiently consumed during heterogeneous nucleation and subsequent fast autocatalytic growth of Co on the "activated" seeds. The mechanistic interpretation of our reaction system, which has been proposed above, is supported by the outcome of pertinent control experiments, whereby $\mathrm{Co}_{2}(\mathrm{CO})_{8}$ is added to seed-loaded reaction flask already containing fresh surfactants (Figure S1 in the Supporting Information). Under the latter conditions, the NRs are made chemically inaccessible as the extra ligand molecules available in the environment can contribute to build up a hardly penetrable organic shell on their surface. Concomitantly, the concentration of Co monomers, whose reactivity toward the seeds is likely to be attenuated upon 
complexation with the surfactants, tends to increase, inevitably leading to independent generation of free-standing, highly stabilized Co NCs after some time. Similar deleterious effects are induced by using exceedingly high decomposition temperatures $\left(>245^{\circ} \mathrm{C}\right)$ and/or performing fast precursor addition to the seeds, which can, in fact, accelerate reaching high supersaturation levels in the solution. Under such conditions, homogeneous metal nucleation events can successfully compete for Co monomer utilization with heterogeneous deposition taking place at the seed surfaces (Figure S1 in the Supporting Information)

The rather low availability of surfactants over the entire course of $\mathrm{Co}_{2}(\mathrm{CO})_{8}$ decomposition as well as the probable existence of differently reactive surface sites (e.g., defects and asperities, or regions of imperfect ligand passivation) in the seed population accounts for the moderate size variance with which the decorating Co domains are ultimately achievable, as observed for other HNC systems synthesized by regulating the ligand concentration to judiciously low levels. ${ }^{27,28}$ Regardless, the heterostructures can develop without any significant tendency toward aggregation, and addition of excess OLAC (a good stabilizer for $\mathrm{Co}$ ) just after consumption of the $\mathrm{Co}_{2}(\mathrm{CO})_{8}$ precursor suffices to ensure long-term stabilization of the asobtained HNCs. ${ }^{27,28}$

The formation of HNCs can be more accurately interpreted in terms of surface tension balance. The attainment of inorganic CdSe@CdS-Co junction is energetically convenient, since it allows the high surface energy, which would otherwise characterize a system composed of NRs and homogenously nucleated Co nanocrystals in a quasi surfactant-free liquid environment, to be significantly decreased at a proportionally smaller cost of attained Co-CdS interfacial energy. ${ }^{3,4,27,28}$ The observed growth regime of selective Co "wetting" of the NR substrates, in which the metal segregates in distinct domains at either basal sides only rather than ubiquitously covering the overall seed surface, can originate from the joint effects of the significant interfacial strain between two hexagonal lattices and the shape anisotropy of the seeds. In this regard, the clear preference of Co to be deposited onto the seed apexes could be preliminarily interpreted as resulting from the inherently higher chemical reactivity and/or surface energy and/or by the comparatively more favorable degree of CdS-Co lattice matching achievable at those locations rather than at the sidewalls. ${ }^{1-4}$ On the other hand, any heterogeneous nucleation pathways, whereby facetpreferential ligand adhesion dictates seed site accessibility, should be expected to be less relevant here, since such a mechanism would, in fact, require precise adjustment of the free surfactant concentration to relatively high levels in order to operate efficiently. ${ }^{28,33}$

The selective production of CdSe@CdS-Co HNCs in the exclusive matchstick-like arrangement deserves further discussion. Such peculiar configuration has been revealed at all synthesis stages with no temporary formation of other types of metal-decorated heterostructure intermediates, which rules out any possible involvement of intraparticle ripening pathways, unlike previously assessed for the reaction of gold with cadmium-chalcogenide-based nanostructures, which could drive interconversion between different topologies. ${ }^{22,41}$ In addition, in contrast to what has been found for $\mathrm{Au}$ and $\mathrm{Pt}$ deposition on similar CdSe@CdS NRs, ${ }^{19,22}$ no HNCs with Co domains asymmetrically positioned along the NR section have been detected with appreciable yields, which allows excluding that preferential localization of lattice strain and/or of free carriers in proximity of the region where the CdSe core is buried may direct Co deposition thereon. This interpretation actually agrees with the fact that Co growth in our HNC synthesis is achieved through thermal decomposition of a zerovalent organometallic precursor, on which any influence of free carriers in the semiconductor seeds should be expected to be irrelevant, unless photocatalytic or electrochemical ripening pathways are triggered. ${ }^{19,22}$

On one hand, the matchstick-like topology of HNCs can be explained on the basis of the structural/chemical dissimilarity of the top and bottom basal sides of $c$-axis elongated wurtzite NRs, which is responsible for their unidirectional anisotropic growth along that axis. ${ }^{1-4,6}$ Considering that the seed population contains both regularly rod- and pencil-shaped objects, whereby the sulfur-rich (001)-type basal facet or the high-energy arrowhead apex would correspond to their fastest growing direction, in the respective cases, Co nucleation can be assumed to be preferred at such most reactive tips. ${ }^{6,18,32}$ Hence, it is reasonable to expect that the Co heads in the HNCs should be located diametrically further from region of the CdSe@CdS tail section where the CdSe core is buried (Scheme 1), ${ }^{6}$ as indeed experimentally suggested by our EFTEM analyses (Figure 4). On the other side, passed a certain size threshold, the probability for Co to be deposited at locations other than the tips can be enhanced on NRs with proportionally higher aspect-ratios (Figure S2 in the Supporting Information). Larger seeds offer extended facets, on which surface defects (e.g., structural imperfections, weakly passivated sites) capable to act as lowenergy nucleation centers can occur more frequently.

Another aspect deserving emphasis is that the particular eccentric core@shell structure of the NRs used here to seed Co nucleation and growth should play an additional important role in dictating their peculiar anisotropic reactivity. This is indeed highlighted by the finding that their CdS-only counterparts instead afford multiply Co-decorated heterostructures without any topological selectivity (cf. Figure 1f). A tentative explanation for such behavior could reside in the different permanent dipole moment associated to the CdSe@CdS NRs, which would arise from the presence of the CdSe nanocrystal core buried in the CdS rod-like shell and from the lattice strain induced locally at that region. ${ }^{49}$ Thus, a remarkably modified dipole could promote heterogeneous nucleation of Co onto one basal sides of the CdSe@CdS NRs, however such a directing effect would be significant enough only in low to medium aspect-ratio seeds, as actually observed by us.

3.4. Optical Properties of the HNCs. The optical behavior of room-temperature solutions of nanocrystal samples is illustrated in Figure 5. As a representative case of study, the properties of CdSe@CdS NR seeds (with diameter/length of $\sim 5 \mathrm{~nm} / \sim 25 \mathrm{~nm}$, grown from $3.5 \mathrm{~nm}$ CdSe nanocrystals) are comparatively examined with respect to those exhibited by HNCs derived thereof (tipped with $\sim 5 \mathrm{~nm}$ Co heads) and corresponding NR/Co physical mixtures (i.e., containing the same NRs and separately synthesized $\sim 5 \mathrm{~nm}$ Co nanocrystals at the same $\mathrm{Cd} / \mathrm{Co}$ molar ratio).

The room-temperature absorption spectrum of the unmodified CdSe@CdS NRs (Figure 5a, solid red curve) shows prominent high-energy peaks below $\sim 495 \mathrm{~nm}$ attributable to absorption from CdS, while the less intense, well-resolved, low-energy peaks at around $\sim 550-610 \mathrm{~nm}$ arise from electronic transitions involving holes and electrons confined in the CdSe core. ${ }^{6}$ The relatively small offset between the CdSe and CdS conduction bands allows for slight electron wave function spreading, leading 


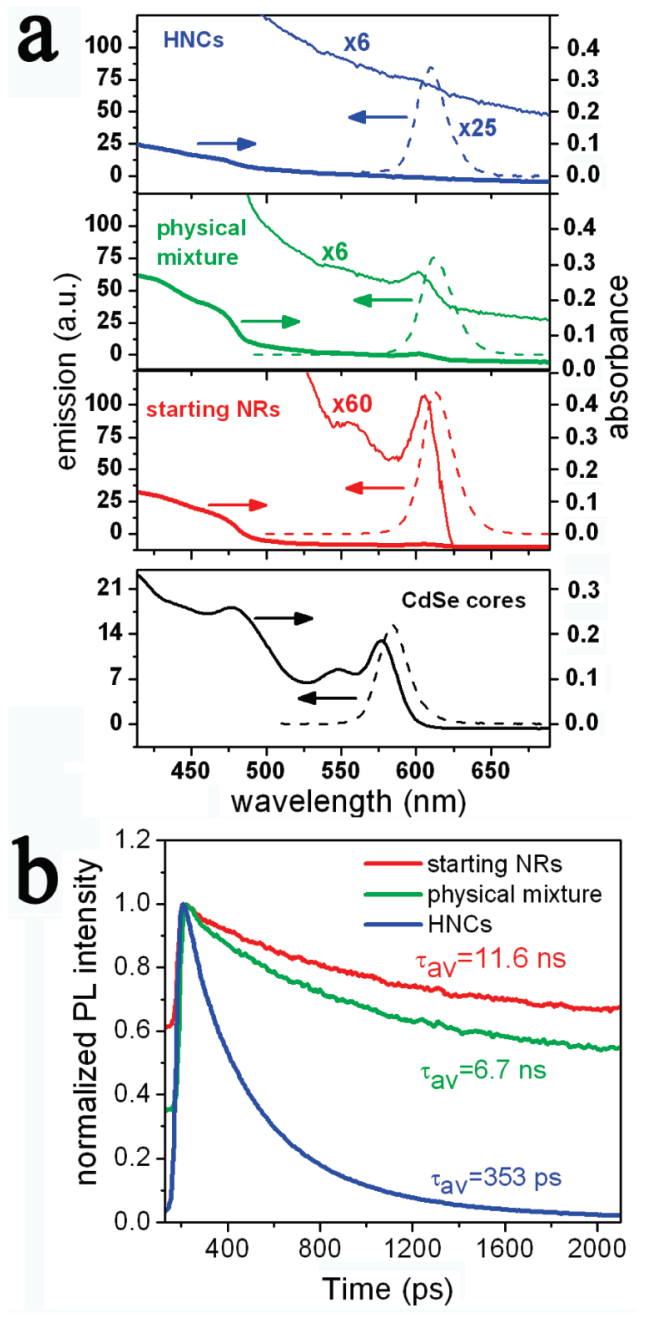

Figure 5. Comparative room-temperature optical characterization of representative solution samples. (a) Steady-state absorption (solid lines, right axis) and PL spectra (dashed lines, left axis) of: CdSe@CdS NRs with diameter/length of $\sim 5 \mathrm{~nm} \sim 25 \mathrm{~nm}$ (red) and corresponding CdSe nanocrystal cores (black); matchstick-like HNCs with $\sim 5 \mathrm{~nm}$ Co tips synthesized from those NRs (blue); physical mixture made of $\sim 5 \mathrm{~nm}$ Co nanocrystals and NRs (green). Note that the cobalt weight percentages in the HNC and mixture samples are identical (about 17\%, according to ICPAES analysis). For the sake of clarity, curves magnified by the factors indicated thereon are also reported, as appropriate. The PL intensities have been scaled so as to reflect the respective QYs (measured exciting at 488 $\mathrm{nm}$ and using Rhodamine $6 \mathrm{G}$ as reference fluorescent dye). (b) Normalized TRPL decay spectra recorded by exciting the samples at $405 \mathrm{~nm}$.

to a small red-shift of the absorption spectrum of the HNCs with respect to that of the CdSe cores (Figure 5a, solid black curve). ${ }^{6}$ The photoluminescence (PL) spectrum (Figure 5a, dashed red curve) exhibits a single sharp peak arising from bandedge recombination, which is accompanied by a small nonresonant Stokes shift similar to that associated to the CdSe cores. All of these features reflect the size and shape homogeneity of the seeds and low interdiffusion between the CdS and CdSe components. ${ }^{6}$

By comparison, major differences characterize the optical properties of the other two samples. In both cases, the contribution from $d$ - $d$ transitions in the Co component leads to an obviously increased absorbance over the whole visible range, with a prominent tail extending beyond the red side of the original NR absorption onset. Notably, the absorption spectrum of the HNCs shows an almost complete dampening of the pristine low-energy exciton features (Figure 5a, blue solid curve), which can not be reproduced with a linear combination of the absorbance of the free NRs and Co nanocrystals (Figure S5 in the Supporting Information), unlike the case of the NR/ Co mixture (Figure 5a, green solid curve). These facts suggest that the semiconductor and metal electronic states are strongly coupled and/or overlapped as a consequence of the direct bonding interfaces attainted between the two materials. ${ }^{17,22}$ Such effects, which are much more pronounced than those reported for bothCo@CdSe core@shell and Co-CdSe-Co dumbbell-like heterostructures (in the latter cases, the exciton signature could be unambiguously identified, even more clearly than for our physical mixture samples), ${ }^{23,24}$ represent a distinctive hint for the good electronic communication established across our metalsemiconductor heterostructures. The small blue shift of $\sim 2-4$ $\mathrm{nm}$, detected both for the absorption onset and the PL peak of the HNCs, can be attributed to the shrinking of the NRs upon Co growth, as mentioned earlier. Most notably, in contrast to their Au-decorated counterparts reported previously, ${ }^{17,22}$ the present Co-tipped CdSe@CdS NRs exhibit appreciable emission intensity. For the specific HNCs concerned in Figure 5, the PL quantum yield (QY) is found to be of about 3\% (with respect to $25 \%$ and $40 \%$ exhibited by the corresponding physical mixture and starting NRs alone, respectively), which is comparable to the highest QY ever reported for semiconductor/ magnetic metal heterostructures. ${ }^{24,29,30,34,40}$

In order to determine the role of the cobalt domain on the relaxation dynamics of the heterostructures, time-resolved PL (TRPL) spectroscopy measurements have been performed. Figure $5 \mathrm{~b}$ reports the TRPL curves relative to the three samples concerned. As commonly observed for colloidal nanocrystals, the recorded decays can be well fitted to biexponential functions (with components featured by $\tau_{1}$ and $\tau_{2}$ lifetime constants, as shown in Figure S6 of the Supporting Information). To the purpose of our study, it is convenient to consider the average radiative lifetime, $\left\langle\tau_{\mathrm{av}}\right\rangle$, referred to as the time at which the PL signal is reduced by a $1 / e$ factor. The starting NRs typically decay with $\left\langle\tau_{\mathrm{av}}\right\rangle_{\mathrm{NR}} \approx 11.6 \mathrm{~ns}$, as assessed previously for NRs with similar aspect ratios. ${ }^{6,49}$ The NR/Co mixture exhibits a slightly reduced lifetime of $\left\langle\tau_{\mathrm{av}}\right\rangle_{\mathrm{mix}} \approx 6.7 \mathrm{~ns}$, which suggests the activation of some nonradiative decay channels, like energy transfer from the semiconductor to the metal nanocrystals. ${ }^{50}$ Despite such processes can be expected to lead to a strong luminescence quenching, their effects are likely to be here attenuated due to the relatively large interparticle distances in diluted nanocrystal solutions. In contrast, the PL signal for the Co-tipped CdSe@CdS HNCs decays very rapidly with $\left\langle\tau_{\text {av }}\right\rangle_{\mathrm{HNC}}$ $\approx 353 \mathrm{ps}$, which is much faster than what has been found for Co-CdSe-Co nanodumbbells. ${ }^{23}$ Such a remarkable $\left\langle\tau_{\text {av }}\right\rangle$ shortening can hardly be ascribed to creation of defects and/or to slight size shrinking that their CdSe@CdS seed components undergo during the course of heterostructure formation in the liquid phase. In fact, the PL decay measured for CdSe@CdS NRs thermally treated in the absence of the cobalt precursor is found to be almost identical to that of the freshly prepared NRs $\left(\left\langle\tau_{\text {av }}\right\rangle\right.$ $\approx 12 \mathrm{~ns})$. Since any involvement of energy transfer pathways could account for only a minor reduction in $\left\langle\tau_{\mathrm{av}}\right\rangle$, a plausible explanation for the PL decay dynamics of the HNCs could invoke occurrence of transfer of the electrons slightly delocalized across the photoexcited semiconductor section toward the metallic domain. This process can be safely assumed to be the main responsible for the observed PL quenching. The extraordinary extent to which the PL lifetime is reduced for Co-tipped CdSe@CdS NRs points to occurrence of an extremely more 

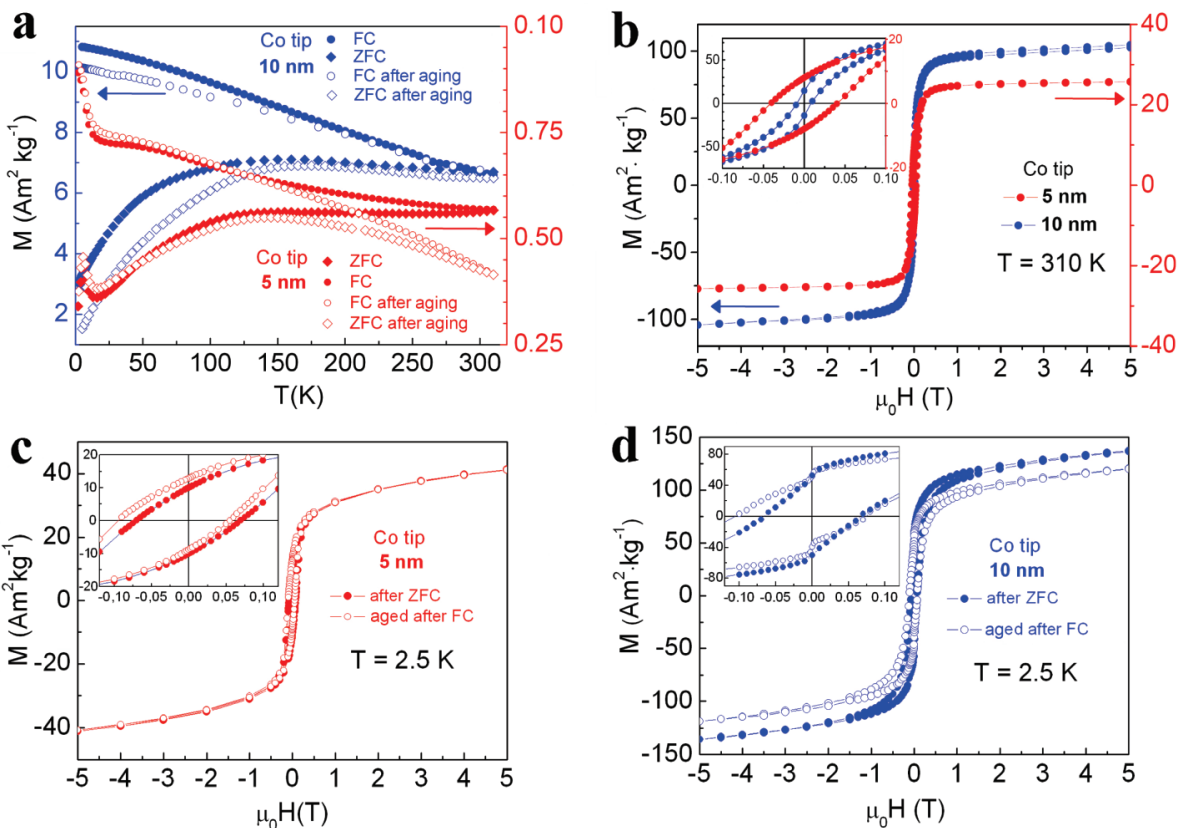

Figure 6. Comparative magnetic characterization of representative powder samples of matchstick-like HNCs made of a $\sim 5 \mathrm{~nm} \times \sim 25 \mathrm{~nm}$ CdSe@CdS tail section and equipped with either $\sim 5 \mathrm{~nm}$ (red curves) or $\sim 10 \mathrm{~nm}$ Co tips (blue curves), respectively. The magnetic data were normalized to the actual Co weight percentages in the samples, which were found to be about $18 \%$ and $35 \%$, respectively, according to ICP-AES analysis. (a) Temperature-dependent zero-field cooled (ZFC) and field-cooled (FC) magnetization curves measured for the as-synthesized (full symbols) and aged samples (i.e., after 3-month storage under $\mathrm{N}_{2}$ in a glovebox and subsequent 24-h exposition to air (empty symbols). The data relative to the $\sim 5 \mathrm{~nm}$ and $\sim 10 \mathrm{~nm}$ Co-tipped heterostructures refer to the right and left magnetization axis, respectively. The ZFC magnetization curves were measured by cooling the samples under a zero magnetic field and then increasing the temperature under a $2.5 \mathrm{mT}$ applied field, while the FC curves were recorded after cooling the samples in a small magnetic field of $2.5 \mathrm{mT}$, respectively. (b) Room-temperature hysteresis loops for the as-prepared samples. (c, d) Hysteresis loops recorded at $2.5 \mathrm{~K}$ for the HNCs equipped with $\sim 5 \mathrm{~nm}$ and $\sim 10 \mathrm{~nm}$ Co tips, respectively. The loops were measured for the as-synthesized samples after a ZFC procedure (full symbols) and for the corresponding aged samples after applying a FC procedure from $300 \mathrm{~K}$ in a $5 \mathrm{~T}$ magnetic field (empty symbols). In the respective insets, magnifications of the low-field loop regions are shown. (Note that the hysteresis cycle for the HNCs equipped with $\sim 5 \mathrm{~nm}$ Co tips was measured by pressing the sample in a pellet in order to prevent nanocrystal reorientation along the external field applied. Differently, the loop measurement for HNCs equipped with $\sim 10 \mathrm{~nm}$ Co tips was recorded on "free" nanocrystal powder. For the latter case, the kinks occurring in the low-field loop region indeed indicate partial reorientation of the nanostructures.)

efficient electron transfer than in their CdSe-only based counterparts. $^{23}$ This process should indeed be greatly facilitated by the energetically favorable band alignment, whereby the Fermi level of Co is indeed positioned well below the conduction band edge of the semiconductor section, ${ }^{17,22,24,22,23}$ as well as by the good structural quality of the shared heterointerfaces.

Ultimately, the appreciable PL properties shown by our metalsemiconductor HNCs may be traced to the following favorable conditions: first, the inherently high PL QY exhibited by the starting CdSe@CdS seeds, relative to that offered by CdSe- or CdS-only NRs, which can satisfactorily compensate for PL quenching; ${ }^{7,17,22,41}$ and, second, the likely reduced extent of mixing between the electronic states of CdSe@CdS and those of $\mathrm{Co}$, with respect to the coupling attained in their $\mathrm{CdSe}-\mathrm{Co}$ system counterparts. ${ }^{23,24}$ Finally, it is worth mentioning that the PL of the HNCs is found to be practically unaltered even after prolonged exposure (up to 3 days) of the heterostructure solutions to ambient atmosphere. Under air, Co starts to be converted to $\mathrm{Co}_{x} \mathrm{O}_{y}$ producing a thin oxide shell that develops at the expense of the metal domain size, until a limiting oxide thickness is reached, at which the oxidation process practically self-arrests. $^{3,27,28,45}$ A large heterojunction between CdS and metallic Co is, however, maintained, which explains why the impact of the metal on the optical properties of the CdSe@CdS section remains practically unchanged.

3.5. Magnetic Characterization of the HNCs. The magnetic performances of the newly synthesized heterostructures have been investigated by superconducting quantum interference device (SQUID) magnetometry. The impact of Co-CdSe@CdS heterojunction formation on the resulting properties can be clearly appreciated from the representative case of study in Figure 6, where the magnetic behavior of HNCs grown from the same CdSe@CdS NRs and carrying Co domains of $\sim 5 \mathrm{~nm}$ and $\sim 10 \mathrm{~nm}$, is summarized. Corresponding Co/NR physical mixtures made of similar NR seeds and unbound Co nanocrystals (in which the softer $\varepsilon$-structure appears to be prevalent) at identical $\mathrm{Cd}$ :Co molar ratio have been comparatively examined (Figures S7-S8 and Table S1 in the Supporting Information). The relevant magnetic parameters are summarized in Table 1.

The temperature dependence of the zero-field cooled (ZFC) and field-cooled (FC) magnetization, $M$, (Figure 6a, solid symbols) reveals that the HNCs are characterized by rather broad distributions of energy barriers, regardless of the Co size. The ZFC maxima that correspond, at a first approximation, to the average blocking temperature $\left(T_{\mathrm{B}}\right)$, are found at $150-200 \mathrm{~K}$ for both samples. Interestingly, the HNCs exhibit thermal irreversibility already at $310 \mathrm{~K}$ (Figure 6b), as corroborated by the hysteretic behavior of $M$ as a function of the applied field, $H$, which reveals coercive fields, $H_{\mathrm{C}}$, of about $9.0-40.0 \mathrm{mT}$ (Tab. 1). These results clearly demonstrate that a fraction of the HNCs is blocked already at $310 \mathrm{~K}$. In contrast, the corresponding NR/Co mixtures display a superparamagnetic behavior under ambient conditions (as confirmed by the absence of coercivity in the $M$ vs $H$ cycles recorded at $310 \mathrm{~K}$ ) and exhibit significantly narrower energy barrier distributions with $T_{\mathrm{B}}$ values lying in the $30-50 \mathrm{~K}$ range, which resembles the behavior of 
Table 1. Magnetic Parameters Measured for the As-Synthesized and Aged HNC Samples Reported in Figure $6^{a}$

\begin{tabular}{|c|c|c|c|c|c|c|c|c|c|}
\hline HNC: Co size & aging conditions & ZFC or FC & $H_{c}^{b}(\mathrm{mT})$ & $M_{5 \mathrm{~T}}(\mathrm{emu} / \mathrm{g})$ & $M_{\mathrm{s}}(\mathrm{emu} / \mathrm{g})$ & $M_{\mathrm{f}} / M_{5 T}$ & $M_{(50 \mathrm{kOe})} / M_{\mathrm{s}}$ & $H_{E B}{ }^{c}(\mathrm{mT})$ & $T(\mathrm{~K})$ \\
\hline \multirow[t]{3}{*}{$5 \mathrm{~nm}$} & as-prepared & $\mathrm{ZFC}$ & 40 & 25.7 & 26.4 & 0.31 & 0.97 & - & 310 \\
\hline & as-prepared & $\mathrm{ZFC}$ & 70 & 41.2 & 465 & 0.24 & 0.89 & - & 2.5 \\
\hline & 3-month stored +24 -h air exposed & $\mathrm{ZFC}$ & 70 & 41.2 & 46.5 & 0.24 & 0.89 & - & 2.5 \\
\hline \multirow[t]{3}{*}{$10 \mathrm{~nm}$} & as-prepared & $\mathrm{ZFC}$ & 9 & 104.7 & 110.2 & 0.14 & 0.95 & - & 310 \\
\hline & as prepared & $\mathrm{ZFC}$ & 66 & 136.8 & 148.4 & 0.40 & 0.92 & - & 2.5 \\
\hline & 3-month stored & FC & $77.5^{b}$ & 132.0 & 146.5 & 0.38 & 0.90 & 10.5 & 2.5 \\
\hline
\end{tabular}

${ }^{a} H_{\mathrm{C}}=$ coercive field; $M_{5 \mathrm{~T}}=$ magnetization value measured at the largest measuring field of $5 \mathrm{~T} ; M_{\mathrm{S}}=$ saturation magnetization derived upon extrapolating the $M$ values for $1 / H \rightarrow 0 ; M_{\mathrm{r}} / M_{\mathrm{s}}=$ reduced remnant magnetization; $T=$ temperature at which the hysteresis loop was measured. ${ }^{b}$ For sample with exchange bias $H_{\mathrm{c}}$ was evaluated as $H_{\mathrm{c}}^{\text {bias }}=\left(H_{\mathrm{c}}^{+}-\mathrm{H}_{\mathrm{c}}{ }^{-}\right) / 2$, where $H_{\mathrm{c}}^{+}$and $H_{\mathrm{c}}{ }^{-}$denote the coercive fields in the positive and negative branches. ${ }^{c} H_{\mathrm{EB}}=\left(H_{\mathrm{c}}^{+}+H_{\mathrm{c}}^{-}\right) / 2$.

Co nanocrystals in this size regime ${ }^{43-48}$ (Figures S7-S8 and Table S1 in the Supporting Information).

A more detailed examination of the low-temperature behavior of ZFC/FC curves of the HNC sample carrying the 5-nm Co tips discloses the existence of a non-negligible fraction of a softer cobalt $\mathrm{Co}_{x} \mathrm{O}_{y}$ phase $\left(\mathrm{CoO}\right.$ and/or $\left.\mathrm{Co}_{3} \mathrm{O}_{4}\right)$, likely in the form of a thin surface shell on the metal domains (invisible to low-magnification TEM) (Figure 6a, red solid symbols). This hypothesis is further corroborated by the hysteresis cycle at 2.5 $\mathrm{K}$ (Figure 6c, solid symbols) which displays exceedingly low magnetization values that do not reach saturation at high fields $\left(M_{5 \mathrm{~T}}=41.2 \mathrm{emu} / \mathrm{g}\right)$, along with a coercive field, $H_{\mathrm{C}} \approx 70 \mathrm{mT}$ and low reduced remanence, $M_{\mathrm{R}} / M_{5 \mathrm{~T}} \approx 0.24$. In addition, the loop recorded after a FC procedure in the presence of a small (positive) magnetic field (data not shown) exhibits a small shift along the field axis toward negative values by an exchange bias (EB) field $\left(H_{\mathrm{EB}}=4 \mathrm{mT}\right)$, which manifests exchange coupling between the ferromagnetic $\mathrm{Co}$ and antiferromagnetic $\mathrm{Co}_{x} \mathrm{O}_{y}$ phases. ${ }^{45,51,52,55}$ On the other side, no evidence for the presence of a $\mathrm{Co}_{x} \mathrm{O}_{y}$ contaminating can be inferred for the HNCs bearing 10-nm Co tips. At $2.5 \mathrm{~K}$ (Figure 6d, solid symbols) such sample indeed exhibits increased coercivity $\left(H_{\mathrm{C}} \approx 67.0 \mathrm{mT}\right)$, much larger magnetization $\left(M_{5 \mathrm{~T}} \approx 137 \mathrm{emu} / \mathrm{g}\right)$ approaching the value expected for bulk $h c p$-Co $(163.1 \mathrm{emu} / \mathrm{g})$, reduced remanence close to the theoretical value of 0.50 (which corresponds to the complete blocking of randomly oriented uniaxial single-domain nanocrystals), and hysteresis curve tending to saturation at high fields (note that these values can be slightly affected by a partial reorientation of the nanocrystals).

The existence of a native oxide surface shell on the Co domains of the as-synthesized samples can be easily explained on the basis of the strong susceptibility of this metal toward

(51) Nogués, J.; Sort, J.; Langlais, V.; Skumryev, V.; Suriñach, S.; Muñoz, J. S.; Baró, M. D. Phys. Rep. 2005, 422, 65-117.

(52) Iglesias, O.; Labarta, A.; Batlle, X. J. Nanosci. Nanotechnol. 2008, 8, $2761-2780$.

(53) Batlle, X.; Labarta, A. J. Phys. D: Appl. Phys. 2002, 35, R15-R42.

(54) (a) Néel, L. Ann. Geophys. 1949, 5, 99-136. (b) Néel, L. Rev. Mod. Phys. 1953, 25, 293-295.

(55) (a) Skumryevù, V.; Stoyanov, S.; Zhang, Y.; Hadjipanayis, G.; Givord, D.; Nogués, J. Nature 2003, 423, 850-853. (b) Nogues, J.; Skumryev, V.; Sort, J.; Stoyanov, S.; Givord, D. Phys. Rev. Lett. 2006, 97, 157203.

(56) Egerton, R. F. Ultramicroscopy 2007, 107, 575-586.

(57) Brydson, R. Electron Energy Loss Spectroscopy; BIOS Scientific: Oxford, U. K., 2001.

(58) Deka, S.; Quarta, A.; Lupo, M. G.; Falqui, A.; Boninelli, S.; Giannini, C.; Morello, G.; De Giorgi, M.; Lanzani, G.; Spinella, C.; Cingolani, R.; Pellegrino, T.; Manna, L. J. Am. Chem. Soc. 2009, 131, 29482958.

(59) Stuczynski, S. M.; Kwon, Y. U.; Steigerwald, M. L. J. Organomet. Chem. 1993, 449, 167-172. attack of oxygen traces already during its solution-phase growth under nominally air-free conditions ${ }^{45}$ (serendipitous oxidation of the Co tips may additionally occur during post-synthesis processing and/or manipulation). Due to their higher chemical potential, comparatively smaller Co nanocrystals with high surface-to-volume ratios are indeed expected to be more prone to oxidation, thereby attaining a passivating $\mathrm{Co}_{x} \mathrm{O}_{y}$ shell at the cost of a proportionally more pronounced metal core size shrinking than in their larger counterparts. ${ }^{45}$

In order to further assess the influence of surface $\mathrm{Co}_{x} \mathrm{O}_{y}$ on the magnetic behavior of the HNCs, the dried sample powders have been examined after a 3-month storage period under $\mathrm{N}_{2}$ in a glovebox and subsequently exposed to air for $24 \mathrm{~h}$ under ambient conditions (cf. Table 1). Following this sequence, the temperature dependence of magnetic susceptibilities is found to be only slightly altered (Figure 6a, empty symbols), implying that the original energy barrier distributions in both samples are little affected by surface oxidation. Further details are disclosed by the corresponding hysteresis cycles at $2.5 \mathrm{~K}$. The 5-nm Co tipped HNCs (Figure 6c, empty symbols) basically show almost unchanged magnetization values, however increased $\mathrm{EB}$ ( $H_{\mathrm{EB}}$ from $\sim 4.0 \mathrm{mT}$ to $\sim 20.0 \mathrm{mT}$ after a $\mathrm{FC}$ procedure in a $5 \mathrm{~T}$ field) and coercivity $\left(H_{\mathrm{C}}\right.$ up to $\sim 75.0 \mathrm{mT}$ after a FC procedure). These facts indicate that for such sample with smaller Co domains the native oxide shell has already reached its limiting thickness prior to deliberate air exposure. Similar arguments can be used to explain the behavior of the 10-nm Co-tipped HNCs (Figure 6d, empty symbols), for which appreciable $H_{\mathrm{C}}$ and $H_{\mathrm{EB}}$ values, along with the magnetization remaining relatively far from saturation (by about $10 \%$ ), confirm the presence of a contaminating antiferromagnetic phase. Subsequent deliberate exposure to air allows the native $\mathrm{Co}_{x} \mathrm{O}_{y}$ shell to develop further to its maximum thickness, which is indicated by a decrease in magnetization (e.g., $M_{5 \mathrm{~T}}$ falls down to $119 \mathrm{emu} / \mathrm{g}$ ) and a concomitant enhancement in both coercivity and $\mathrm{EB}$ effects $\left(H_{\mathrm{C}}\right.$ and $H_{\mathrm{EB}}$ reach up to $90.5 \mathrm{mT}$ Oe and 16.0 $\mathrm{mT}$, respectively, upon a FC procedure), which do not change any further upon increasingly prolonged storage.

Overall, the magnetization thermal stability at room temperature which distinguishes the present Co-tipped CdSe@CdS NRs is largely unexpected on the basis of the well-documented magnetic behavior of free-standing Co nanocrystals in this dimensional regime arising from finite size and surface effects, ${ }^{43-48,53}$ which has been, in fact, verified to still hold for the NR/Co mixture samples. A rough estimation of the mean anisotropy energy density, $K_{\text {anis }}$, can be obtained using the Néel model, ${ }^{54}$ according to the following expression: 


$$
K_{\text {anis }}=25 k_{\mathrm{B}} T_{\mathrm{B}} / V_{\mathrm{Co}}
$$

where $k_{\mathrm{B}}$ is the Boltzmann constant and $V_{\mathrm{Co}}$ is the Co nanocrystal volume evaluated from TEM measurements. Our data suggest average $K_{\text {anis }}$ values at least larger than $2 * 10^{5} \mathrm{~J} / \mathrm{m}^{3}$ for $\mathrm{Co}$ in the HNCs, which are among the largest ever achieved for colloidal nanoheterostructures combining magnetic and nonmagnetic materials. ${ }^{1-4,23,24,29,30,32,34,36-40}$ Even assuming a dominance of $\varepsilon-\mathrm{Co}$ in the NR/Co mixtures, ${ }^{43-48}$ the magnetic properties of the HNCs cannot be fully justified by invoking the higher magnetocrystalline anisotropy associated with the hcp-phase of the Co domains in the heterostructure and/or the interplay of dipolar interactions (the latter should, in fact, be expected to be of similar strength for both HNCs and their NR/ Co mixtures). Neither ferromagnetic-antiferromagnetic exchange biasing interactions between $\mathrm{Co}$ and $\mathrm{Co}_{x} \mathrm{O}_{y}$ phases in $\mathrm{Co}_{x} \mathrm{O}_{y^{-}}$ passivated Co domains could suffice to provide alone most of the extra anisotropy necessary for thermal stabilization of the magnetization, unlike found for other noncolloidal systems (e.g., for $\mathrm{Co}$ nanoparticles embedded in $\mathrm{CoO}$ matrix). ${ }^{51,55}$ In fact, similar beneficial effects should have been equally observed for their NR/Co mixture counterparts.

On the basis of the above observations, it can be concluded that the room-temperature ferromagnetic-like behavior of the HNCs should inherently correlate with the existence of bonding junctions between $\mathrm{CdS}$ and Co. This is further suggested by the fact that their multiply Co-decorated CdS-only heterostructure counterparts are characterized by analogously unusual magnetic properties (Figure S9 in the Supporting Information). One plausible explanation would ultimately consider the attainment of a strained interfacial region with unique compositionalstructural features (e.g., $\mathrm{Co}_{x} \mathrm{~S}_{y}$ buffer layer ${ }^{59}$ of graded composition), whereby electronic structure modifications and symmetry breaking could unusually influence the magnetic anisotropy term. The attainment of various types of heterojunctions, at which different relative orientations and, hence, bonding relationships can subsist between the $\mathrm{CdS}$ and Co lattices, could contribute to the broadening of the energy barrier distribution, eventually smoothing out the typical size-dependence of the magnetic behavior in otherwise unbound Co nanocrystals. ${ }^{28}$ As the junction area that Co shares with the semiconductor is a relatively large fraction of the metal domain surface, the ultimate impact on magnetic properties can be indeed expected to be relevant, as demonstrated in previous studies on the effects of capping layers ${ }^{47,48}$ and heterointerface formation in other types of Co-based HNCs. ${ }^{24,28}$ The crucial role of specific CdS-Co bonding relationships achieved in the present HNCs emerges clearly by considering that their spherical Co@CdSe core@shell and Co-tipped CdSe nanorod analogues do not block at room temperature unless anisotropically shaped Co domains are introduced in the heterostructures. ${ }^{23,24}$

\section{Summary and Conclusions}

In summary, we have demonstrated a sequential seededgrowth approach to synthesize matchstick-like magneticsemiconductor HNCs that comprise one-sided Co-tipped rodlike section of $\mathrm{CdS}$ embedding a CdSe nanocrystal. The specific topological regime achieved has been found to originate from the peculiar anisotropic reactivity of the noncentrosymmetric CdSe@CdS core@shell nanorods that have been exploited to seed heterogeneous nucleation of Co from an organometallic precursor in a surfactant-free noncoordinating medium. Besides retaining appreciable fluorescence emission in spite of occurrence of semiconductor-to-metal electron transfer, the newly developed HNCs exhibit unusual ferromagnetic-like properties at room temperature as a consequence of the direct bonding junctions between Co and CdS. These bifunctional magneticfluorescent Co-tipped CdSe@CdS nanorod heterostructures could find numerous applications in visible-light-driven photocatalysts (e.g., as magnetically recoverable catalysts), in the biomedical field (e.g., in analytical separations, drug delivery, dual-mode imaging), and in spintronics (e.g., in spin-polarized light-emitting diodes or spin transistors). Overall, our study suggests that an increased level of synthetic sophistication and topological selectivity in HNC preparation could ultimately be reachable by means of seeded-growth techniques that exploit nanocrystal seeds with suitably engineered compositional and structural parameters. It can be also envisioned that the creation of interface junctions between magnetic and nonmagnetic nanoscale materials could be viable as a new tool to generate unprecedented magnetic properties that are distinct from those of single-component nanocrystals and their physical mixtures.

Acknowledgment. The Italian Ministry of Research (Contract No. RBIN048TSE) is acknowledged. We thank Angela Fiore and Rosanna Mastria for providing samples of CdS nanorods and for many helpful discussions.

Supporting Information Available: TEM examples of control syntheses performed in the presence of surfactants and at high precursor injection rates. TEM example of HNCs grown from high aspect-ratio CdSe@CdS NRs. Time-dependent TEM studies on HNC formation. TEM and XRD analysis of Co nanocrystals synthesized in the absence of NR seeds. UV-vis absorption spectra of Co nanocrystals. Fits of the TRPL decays. Magnetic data on Co nanocrystal/CdSe@CdS NR physical mixtures and on HNCs grown from CdS-only NRs. Complete ref 6 . This material is available free of charge via the Internet at http://pubs.acs.org.

JA904493C 